\title{
Neural Network Algorithm for Stabilizing Mechanized Systems
}

\author{
SHMAKOVA ELENA G., FILORETOVA OLGA A., NIKOLAEVA OLGA M., \\ VASILKIN DENIS P. \\ Department of Information Systems in Chemical Technology \\ Lomonosov Institute of Fine Chemical Technological, MIREA-Russian Technological University \\ Vernad-sky Ave 86, Moscow, 119571 \\ RUSSIA
}

\begin{abstract}
The article describes an experimental model of stabilization of a mechanized system. The following are shown: a skate; an element of the program code; an algorithm for stabilizing a proportional-integraldifferential controller (PID). The experimental model uses the calculation and adjustment of the regulator according to the Ziegler-Nichols method. For the case of applying the neural network approach to the search for equilibrium, the Hopfield neural network is used. The technology of calculating the balancing of the values of the coefficients: proportional, integral, differential components are described. The design of the rolling system is described. The experimental model is designed to identify the balancing range of the rolling system of smalldiameter balls. The experimental module balances the ball at a distance of 4.5 to $7 \mathrm{~cm}$ (SW-range). The shortcomings of the experimental model of stabilization of the mechanized system are revealed. The analysis of experimental studies of spacecraft stabilization is carried out. It is determined that it is advisable to use the mathematical tools of the sixth-order Butterworth polynomial in the training of a neural network. Complex neural network calculations make it possible to calculate the stabilization coefficients of the spacecraft when the coordinate system does not coincide with the axes of inertia. An overview of the authors ' research on the use of intelligent quality control systems for the production of medicines is given. An overview of neural network solutions for stabilizing the turning angle of high-speed cars is given. The expediency of selecting the stabilization coefficients of a proportional-integral-differential regulator by a trained neural network for various rolling ranges is proved.
\end{abstract}

Key-Words: neural network, stabilization of the rolling system, experimental model, Butterworth polynomial, selection of stabilization coefficients of the proportional-integral-differential regulator.

Received: April 12, 2021. Revised: November 18, 2021. Accepted: December 20, 2021. Published: January 8, 2022.

\section{Introduction}

The stabilization of mechanized systems is realized with the highest possible accuracy. The stabilization of mechanized systems is required for unmanned aerial vehicles, industrial robotics, chemical industry, mechanical engineering, power engineering (control of boiler rooms), mechanics (regulation of servos), high-precision equipment of the space industry. The stabilization of mechanized systems includes the following processes: design of an automated stabilization system, organizational and technical design, neural network processing of input and output parameter values. The improved stabilization algorithm increases accuracy, safety, and minimizes the probability of errors. The authors develop a neural network software algorithm for stabilization and propose a model of its prototype. The process of developing a stabilization module based on a PID controller is described, the technical features of the module development are identified. To accomplish this task, the authors needed to analyze the available hardware and software implementation tools, identify advantages and disadvantages, develop a neural network algorithm, develop a prototype of the system and test it. The main goal of the experiment is to develop a rolling system for small-diameter metal balls on a 3D printer. To develop and apply an algorithm for stabilizing the rolling system by combining technical capabilities: a microcontroller, a minicomputer and a neural network algorithm. The computing device is a proportional-integraldifferential controller. The study shows: a skate; an element of the program code; an algorithm for stabilizing a proportional-integral-differential regulator (PID). The experimental model uses the calculation and adjustment of the regulator according to the Ziegler-Nichols method. For the case of applying a neural network approach to the search for equilibrium, the Hopfield neural network is used. The technology of calculating the balancing of the values of the coefficients: proportional, integral, differential components is described. The 
design of the rolling system is described. The experimental model was developed in order to identify the balancing range of the rolling system of small diameter balls. The experimental module balances the ball at a distance of 4.5 to $7 \mathrm{~cm}$ (SWrange). The shortcomings of the experimental model of mechanized system stabilization are revealed.

The advantage of the work is the design on a $3 \mathrm{D}$ printer of an experimental model of the rolling system (Fig.6.- Fig.8) and the development of a program for the Arduino $\mathrm{C}$ microcontroller and a proportional-integral-differential controller.

\section{Devices for Controlling the Parameters of the Stabilization Process}

Most prototypes are developed using microelectronic elements, microcontrollers, singleboard computers. Unicameral computers use operating system programs. Combining the technical capabilities of a microcontroller, a minicomputer and a neural network algorithm, in total, give a synergistic effect. Arduino boards read data from sensors, output motor signals, LED signals, network signal data. The software for writing code (sketch) is free, multiplatform. The development of a neural network algorithm uses the theory of microelectronics, programming, design, modeling of computer technology, analog-digital data processing. A minicomputer based on a Raspberry Pi microprocessor (memory, storage, graphics driver, connection connectors) and an Arduino microcontroller (central memory, RAM and permanent storage devices) provide input and output signals. All signals are processed by the algorithm. The Arduino clock frequency is $16 \mathrm{MHz}$. The clock frequency of the Raspberry Pi is $1.2 \mathrm{GHz}$. Raspberry Pi is convenient for developing software applications using the Python programming language. Arduino is convenient for controlling: LEDs; sensors; the position of the motors; the coordinates of the motor movement; switching buttons. Intelligent (neural network) digital control of input and output parameters of mechanized systems is the main task of stabilization. To create a prototype, special functions are required, engine drivers, an Ethernet connection, an SD card reader, Wi-Fi, touch screens, cameras, GPS, RGB panels, etc. The power requirements of the Raspberry $\mathrm{Pi}$ and Arduino are different. Despite the fact that both are powered by USB (micro-USB or USB Type C for Raspberry Pi and USB Type B for Arduino), Raspberry Pi requires more current than Arduino. To work with the Raspberry $\mathrm{Pi}$, a power adapter is required, to work with the Arduino, it is enough to simply connect to a computer via a USB port. Interrupting the power supply of the Raspberry $\mathrm{Pi}$ causes damage to the hardware, software or applications. In the case of Arduino, when the power is turned off, the microcontroller restarts. Raspberry Pi must be properly connected and properly turned off. It is used by the Arduino IDE for code development. Raspberry $\mathrm{Pi}$ use the languages Python IDLE, Eclipse IDE, supported by Linux. Raspberry Pi programming is carried out using a terminal, a text editor is used for this. The Arduino open source hardware and software files are used. Raspberry Pi is not open source software. Important advantages of Arduino are: crossplatform, practical IDE architecture, open source code, additional ability to use AVR-C code.

2.1 Sketch, a sketch of the program code

All sketches and sketches of the program are saved in a special extension - .ino. The message area displays: feedback on saving and exporting; current errors. The console displays the text output of the Arduino software (IDE). Includes full error messages and other information. The configured card and serial port are displayed in the lower right corner of the window. The toolbar buttons allow you to check and load programs, create, open and save thumbnails, and open a serial monitor (Fig.1).

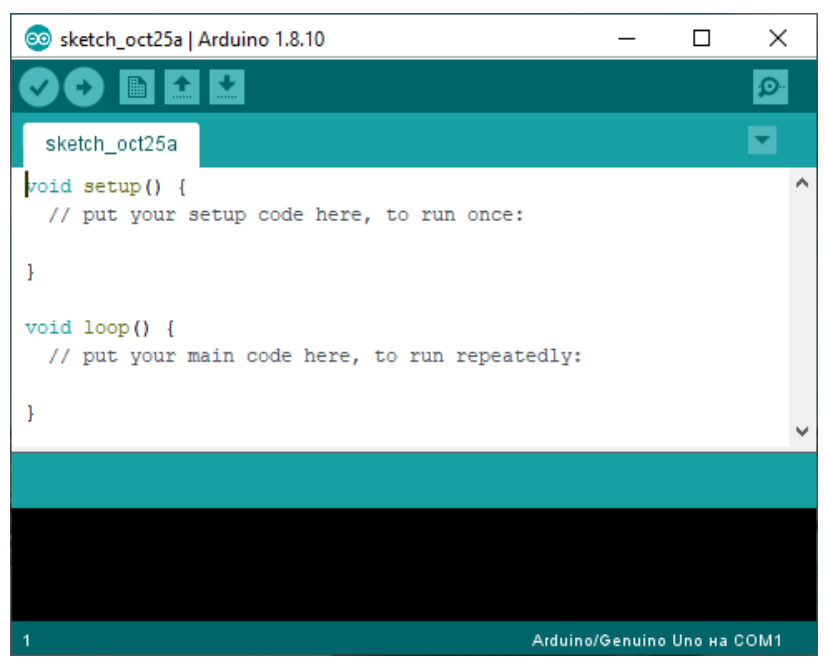

Fig.1: Arduino IDE Interface

The Arduino software (IDE) uses the concept of a notepad: a standard place to store programs (or sketches). Thumbnails in the album can be opened from the "File" menu, "Drawing Album" or using the "Open" button on the toolbar. When you first start the Arduino software, a drawing album catalog is automatically created. Sketch Features:

1. connecting the code from an external file;

2. loading the sketch through the programmer directly into the microcontroller; 
3. export the binary file and store the compiled file.

The output panel displays the following information: about the successful compilation of the code, in addition, an error message.

\subsubsection{Proportional-integral-Differential Controller (PID)}

The PID controller is a device for controlling the process parameters: pressure, fuel consumption, temperature, speed. The PID controller is used for small aircraft, regulates the control of the servo drive. The PID controller is involved in stabilizing the position of the flying object in space. In the controller, the feedback device of the control loop is used to regulate variable processes. In a closed-loop controller, a control loop feedback device is used to maintain the required output signal value. The closed system of the PID controller includes a feedback control system. The system evaluates the variable feedback value. A fixed point of error signal generation is used. The output values change. The error is approaching zero, reaching zero.

When the value falls below a fixed point, the controller turns off. The controller is turned off if the value is higher than the fixed value. The output signal of the PID controller is unstable, fluctuates within a fixed point. The operation of the controller is represented by a block diagram (Fig. 2).

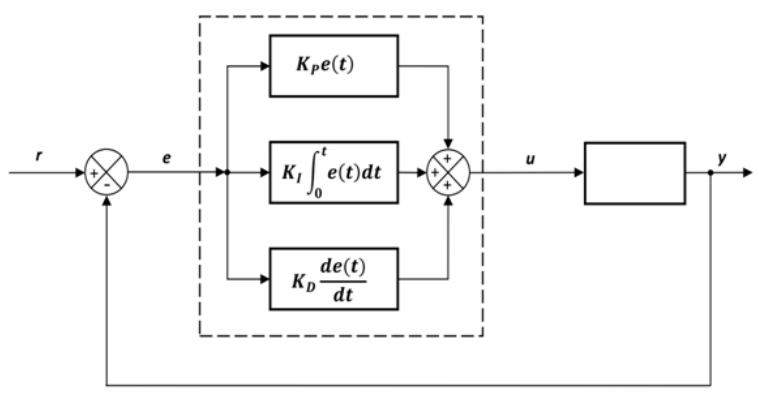

Fig. 2: Block diagram of the PID controller.

\subsubsection{Two-position Control of the Regulator with Feedback}

The two-position controller moves the controlled variable from the OFF position to the ON position, depending on the variable. The PID controller supports the output signal in such a way that there is a zero error between the process variable and the set value (in the case of performing feedback operations) (Fig. 3).

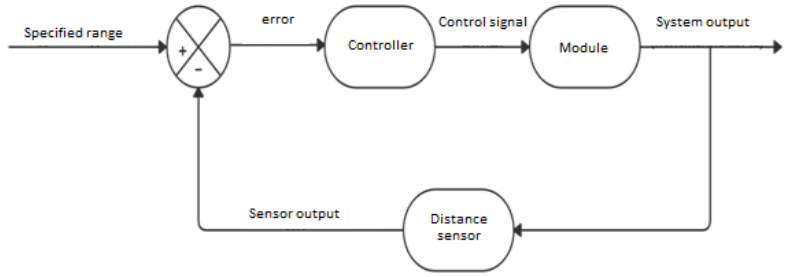

Fig. 3: Block diagram of the experimental model PID controller.

\section{The Algorithm of Stabilization using the PID Controller}

To develop a stabilization algorithm, we will determine the components of the experimental setup of the stabilization system:

1. Servo drive;

2. Infrared sensor (tracking the movement of the ball);

3. Swing (swinging the ball);

4. Arduino microcontroller (swing control, sensor data processing, controller start-up, servo control);

5. Potentiometer (a measuring device for comparing two voltage values in real time).

The experiment uses metal balls that swing on a swing (Fig. 4).

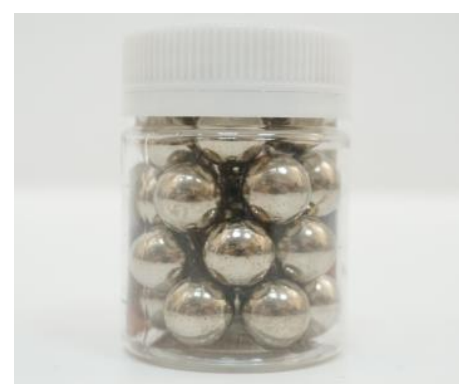

Fig. 4: Metal balls with a diameter of $12 \mathrm{~mm}$.

The main task of stabilizing the system is to balance the values of the coefficients: proportional integral, differential components.

The stabilization algorithm is defined by the following procedure:

1. power supply to the circuit blocks

2. data linearization (from Lat. linearis-linear) is one of the methods of approximate representation of closed nonlinear systems, in which the study of a nonlinear system is replaced by the analysis of a linear system;

3. calculations of the two-position control of the regulator with feedback;

4. data output to the servo; 
5. at the beginning: reading data from the sensor, then the cycle enters the data linearization (Figure

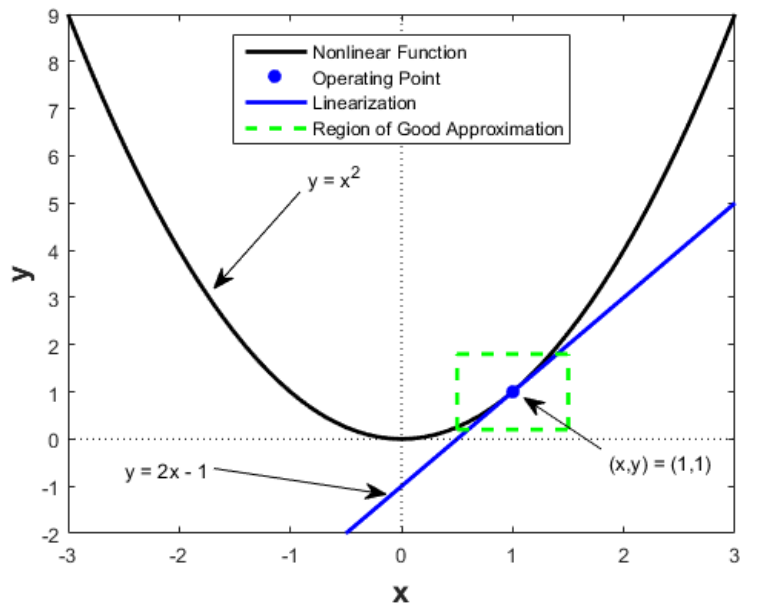

Fig. 5: Linearization.

Linearization with feedback is a way to bring the system to a form where there is an external parameter of the control action. In this case, the nonlinear system becomes linear, and external control is provided for stabilizing and controlling the remaining linear part of the system (Fig. 5).

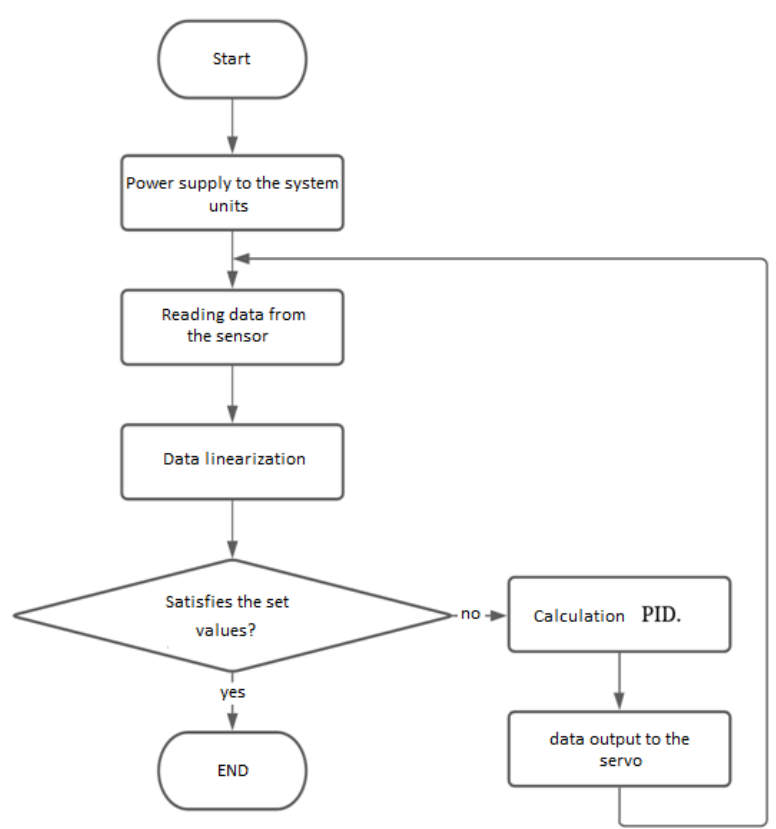

Fig. 6: The algorithm of stabilization of the experimental model

The Ziegler-Nichols method is a method of automatic calculation and adjustment of regulators. This method is empirical and is based on the use of data obtained experimentally on a real object. For the case of applying the neural network approach to the search for equilibrium, the Hopfield neural network is used - a fully connected neural network with a symmetric matrix of connections. In the process of operation, the dynamics of such networks converges (converges) to one of the equilibrium positions. These equilibrium positions are determined in advance in the learning process, they are the local minimum of a functional called the network energy (in the simplest case, the local minima of a negatively defined quadratic form on an n-dimensional cube) [3]. Such a network can be used as an auto-associative memory. The trained neural network selects the final values of the coefficients: proportional integral, differential components(Fig. 6),(Fig.7).

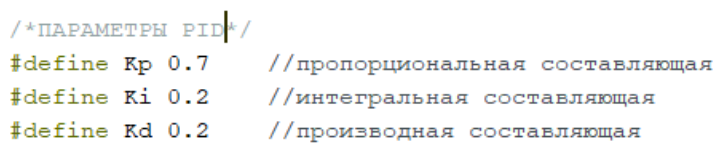

Fig. 7: The final values of the coefficients

The applied element base of the experimental model:

1. Arduino Uno Board

2. Hitec HS-422 Analog servo drive

3. GP2Y0A41SK0F distance sensor

4. Potentiometer

5. Rocking Chair (Rolling system) Actobotics Single Servo Arm

6. Metal ball

The design of the experimental rolling system was carried out in Autodesk 3ds Max. Design of rolling module parts: base (Fig.8), gutter (Fig.9), bracket (Fig. 10).

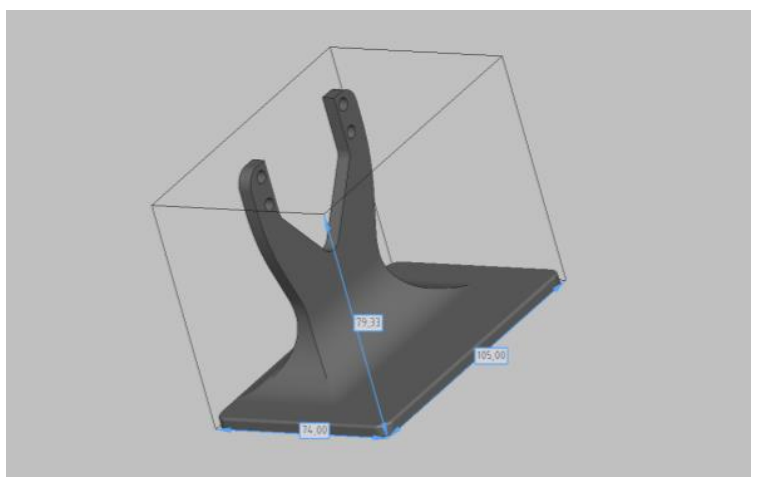

Fig. 8: Module base 


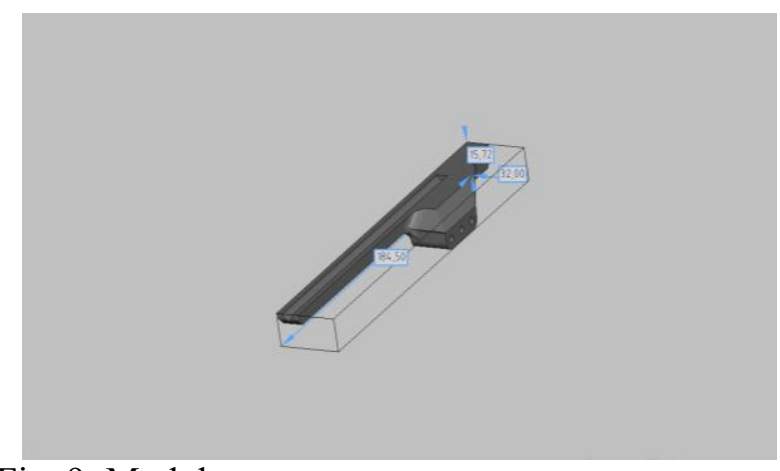

Fig. 9: Module gutter

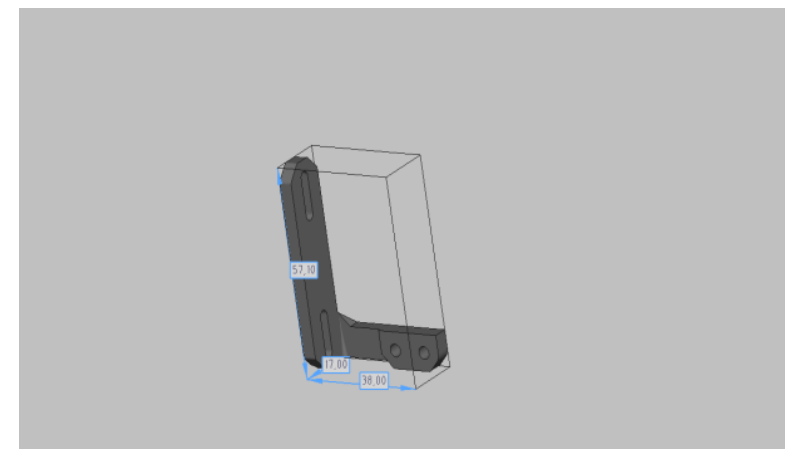

Fig. 10: Module bracket

The experimental model was developed in order to identify the range of ball rolling balancing. The implementation of the software part of the experimental module was carried out in the Arduino $\mathrm{C}$ programming language (the $\mathrm{C}++$ programming language connected to the Wiring framework) and the Arduino IDE development environment. To program the board on the Arduino microcontroller, a special development environment of the Arduino IDE was chosen. This software is open source and cross-platform, available for use on several operating systems (Arduino IDE is available for use on Windows, Mac OS, and Linux). The programming language is Wiring, the syntax is similar to such programming languages as $\mathrm{C}$ and $\mathrm{C}++$, the operating system is Windows. Before creating a sketch, you need to perform the following actions:

1. Connect the board via the USB port;

2. Specify the port in the Arduino IDE;

3. Develop a sketch.

Next, the sketch is uploaded to the board. Development of an algorithm for the operation of the software component:

1. connect the Servo.h library to control the servo;

2. determine the initial position of the servo, specify the values of the PID controller;
3. assignment and initialization of variables, determination of the numbers used in the operation of pins.

4. in the setup function, a serial connection is initiated, the data transfer rate in bits/s is set, and the port number from which the drive will be controlled is specified.

5. in the cycle, $\Delta \mathrm{t}$ is written, data from the distance sensor is read, we get rid of the" noise " and linearize the received data.

6. we count the PID, output the data to the servo.

If the ball position does not meet the set values, the cycle is repeated, this happens until the set values are reached.

The experimental module balances the ball well at a distance of 4.5 to $7 \mathrm{~cm}$. This is the SW range, not the real range, the sensor is not $100 \%$ calibrated. If you hit the ball, the module compensates for this and returns the ball to the starting point. It also responds to a change in the initial preset position (using a potentiometer). The code is gradually struggling more and more with large ranges. Over time, it compensates for the established errors due to the unevenness of the gutter.

\section{Conclusion}

In conclusion, it should be noted that the experimental model allowed us to determine the stabilization of the system for balancing the ball in the range of 4.5 to $7 \mathrm{~cm}$. This suggests that the Ziegler-Nichols method of calculating and adjusting regulators can be used for rolling systems with a ball diameter of $12 \mathrm{~mm}$.

In the study of the authors Vorobyov V. Yu., Sablina G. V. "Calculation and optimization of the parameters of a discrete PID controller by the Ziegler-Nichols method", the calculation of the parameters of the PID controller was carried out using the Matlab software and the Situlink library. In the study of the authors, it was proved that the Ziegler-Nichols method does not take into account the requirements for the stability margin - this is the main drawback. After calculating the parameters of the controller, manual adjustment is required to improve the quality [4], [5], [6].

The conducted experiment confirms the need to use a neural network approach. The trained neural network selects stabilization coefficients for 
different ball diameters, taking into account the ranges [2].

In the study of the authors Zubov N. E. and others "Stabilization of the orbital orientation of the spacecraft", the mathematical tools of the Butterworth polynomial of the sixth order are given[1]. The calculation of the roots of the polynomial is used in the matrix, which forms the coefficients of the regulator. Complex neural network calculations make it possible to stabilize the spacecraft when the coordinate system does not coincide with the axes of inertia. The neural network method solves the problems of pole placement. Analytical expressions of the feedback matrix of the controller solve the problems of stabilization of the spacecraft's orbital station [7].

In the study of the authors Saikin A.M., Buznikov S. E., Shabanov N. S., Elkin D. S. and others "Mathematical model of the dynamic stabilization system of an unmanned vehicle", a study of dynamic stability stabilization along the course is carried out. Stabilization is necessary for high-speed cars, in case the car exceeds the speed limit [10].

Application of neural network solutions in the study of the authors Kornyushko V. F. and others, the issues of quality management of the chemical and technological process of continuous synthesis of pharmaceutical substances of medicinal compounds in flow microreactors are considered. The necessity of using intelligent control systems for quality control of the production of medicines is proved [8]. Calculations and technologies of the team of authors of the Department of Information Systems in Chemical Technology under the leadership of Shmendel E.V. are used for chemical industries [1]. The study of the authors Khitskov E. A, Veretekhina S. V., Medvedeva A.V., Mnatsakanyan O. L., Shmakova E. G., Kotenev A "Digital transformation of society: Problems entering in the digital economy "examines the issues of building a parallel digital reality. The main tool for building digital reality is trained neural networks [9].

\section{References:}

[1] Shmendel E.V., Kabilova T.O., Morozova N.G., Zenkova M.A., Maslov M.A. Effects of spacers within a series of novel folate-containing lipoconjugates on the targeted delivery of nucleic acids, Journal of Drug Delivery Science and Technology, 2020, 101609 doi.org/10.1016/j.jddst.2020.101609

[2] Zhmud V.A., Sablina, G.V., Trubin,V.G. Dimitrov L.V. Use of bypass channel for feedback control of oscillatory object well-known as difficult one for control, International Siberian conference on control and communications (SIBCON): proc., Moscow, 12-14 May 2016. IEEE, 2016. - $6 \mathrm{p}$

[3] Vorobyev V. Yu., Sablina G. V. Calculation and optimization of parameters of a discrete PID controller by the Ziegler-Nichols method / / Automation and Software Engineering, 2019 1(27) http://www.jurnal.nips.ru

[4] Zhmud, V., Dimitrov, L., Yadrishnikov, O. Calculation of regulators for the problems of mechatronics by means of the numerical optimization method // 20.15.2014 12th International Conference on Actual Problems of Electronic Instrument Engineering, APEIE 2014 Proceedings 7040784, p. 739-744.

[5] Ivoilov A.Y., Zhmud V.A., Trubin V.G, Dimitrov L. V. Detection of unrevealed non-linearities in the layout of the balancing robot /. International Siberian conference on control and communications (SIBCON): proc., Moscow, 12-14 May 2016. Moscow: IEEE, 2016. $-9 \mathrm{p}$.

[6] Zhmud V., Yadrishnikov O., Poloshchuk A., Zavorin A. Modern key technologies in automatics: structures and numerical optimization of regulators. Proceedings - 2012 7th International Forum on Strategic Technology, IFOST 2012 2012. C. 6357804.

[7] Zubov N. V., Lapin A.V., Mikrin E. A. Stabilization of the orbital orientation of the spacecraft // Space Engineering and Technologies 3, 2013 pp. 74-81.

[8] Kornyushko Valery F., Nikolaeva Olga M., Panov Alexey V., Biglov Rem R., Kuznetsov Andrei S., 2021 Quality management of the chemicaltechnological process for continuous synthesis of pharmaceutical substances of medicinal compounds in flow micro reactors // MATHEMATICS METHODS AND INFORMATION SYSTEMS IN CHEMICAL TECHNOLOGY Fine Chemical Technologies $=$ Fine Chemical Technologies. ISSN 2686-7575 (Online),16(3):252-266.

[9] Khitskov E.A, Veretekhina S.V., Medvedeva A.V., Mnatsakanyan O.L., Shmakova E.G., Kotenev A, 2017. Digital transformation of society: Problems entering in the digital economy // Eurasian Journal of Analytical Chemistry 12(5) 855-873

[10] Saikin A.M., Buznikov S. E., Shabanov N. S., Elkin D. S. and others "Mathematical model of the dynamic stabilization system of an unmanned vehicle", IOP Conference Series: Materials Science and Engineering, Volume 315, Issue 1, page 012023 (2018). DOI 10.1088/1757-899X/315/1/012023

\section{Creative Commons Attribution License 4.0 (Attribution 4.0 International, CC BY 4.0)}

This article is published under the terms of the Creative Commons Attribution License 4.0 https://creativecommons.org/licenses/by/4.0/deed.en US 